\title{
Smoothing Spline Curves and Surfaces for Sampled Data
}

\author{
Hiroyuki Fujioka ${ }^{1}$, Hiroyuki Kano ${ }^{1}$, Magnus Egerstedt ${ }^{2}$ and Clyde F. Martin ${ }^{3}$ \\ 1) Department of Information Sciences, \\ Tokyo Denki University, Saitama 350-0394, Japan. \\ E-mail: \{fujioka,kano\}@j.dendai.ac.jp \\ 2) School of Electrical and Computer Engineering, \\ Georgia Institute of Technology, Atlanta, GA 30332, USA. \\ E-mail: magnus@ece.gatech.edu \\ 3) Department of Mathematics and Statistics, \\ Texas Tech University, Lubbock, TX 79409, USA. \\ E-mail: martin@math.ttu.edu
}

\begin{abstract}
We consider the problem of designing optimal smoothing spline curves and surfaces for a given set of discrete data. First we derive concise expressions for the optimal solutions using normalized uniform Bsplines as the basis functions. Then, assuming that a set of data in a plane is obtained by sampling some curve with or without noises, we prove that, under certain condition, optimal smoothing splines converge to some limiting curve as the number of data increases. Such a limiting curve is obtained as a functional of given curve to be sampled. The case of surfaces is treated in parallel, and it is shown that the results for the case of curves can be extended to the case of surfaces in a straightforward manner.
\end{abstract}

Key phrases: B-splines, optimal smoothing splines, asymptotic analysis, statistical analysis

\section{Introduction}

The problem of optimal design of approximating or interpolating curves and surfaces for a given set of data arises in various fields of engineering and sciences. In particular, spline functions have been used frequently, and thus have been studied extensively (e.g. [15]). In addition to traditional approaches in spline studies, an approach based on optimal control theory has been employed for generating piecewise polynomial splines as the solutions to a number of optimal control problems $[10,11]$. Moreover, the theory of 'dynamic splines' provides a unified framework for generating various types of spline curves as outputs of linear dynamical systems (e.g. $[16,13,2]$. Also, the authors studied B-spline functions from the viewpoint of optimal control theory [6]. In the problem of designing surfaces by splines $[12,5]$, the surfaces, in most cases, are constructed by resorting to some numerical optimization techniques. Alternative approaches to this problem include the development of appropriate basis functions, e.g. wavelets, and active boúndary control of partial differential equations [8].

When we are given a set of data corrupted by noise, smoothing splines are expected to yield more feasible solutions than interpolating splines. A theoretical issue in this regard is asymptotic and statistical analyses of designed splines when the number of data increases. Such a problem is studied in [3] in a dynamical systems setting, namely for spline curves generated as the output of linear dynamical systems. On the other hand, using B-splines [1] as basis functions is expected to yield simple algorithms for designing curves and surfaces. In particular, using normalized uniform B-splines as the basis functions, we have been studying the problems of constructing optimal interpolating and approximating curves $[6,9,4]$. This approach makes it easier to extend the results for one-dimensional case (i.e. the case of curves) to two-dimensional case (i.e. the case of surfaces) and to even higher dimensions.

The purpose of this paper is to design optimal smoothing spline curves and surfaces, and analyze their properties using normalized uniform B-splines as the basis functions. Assuming that a number of data is given by sampling some curve $f(t)$ with noise, we analyze statistical properties of optimal smoothing splines and derive an expression of the splines as a functional of $f(t)$ when the number tends to infinity. Such design and analysis methods are extended to the case of surfaces. We will see that the expressions for optimal curves and surfaces are concise, enabling us to analyze their properties. Moreover, extensions of the results for curves to the two-dimensional case, namely the surfaces, are straightforward.

For designing curves $x(t)$, we employ normalized, uniform B-spline function $B_{k}(t)$ of degree $k$ as basis functions,

$$
x(t)=\sum_{i=-k}^{m-1} \tau_{i} B_{k}\left(\alpha\left(t-t_{i}\right)\right) .
$$

Here, $m$ is an integer, $\tau_{i} \in \mathbf{R}$ are weighting coefficients 
called control points, and $\alpha(>0)$ is a constant for scaling the interval between equally-spaced knot points $t_{i}$ with

$$
t_{i+1}-t_{i}=\frac{1}{\alpha}
$$

Then the $x(t)$ formed in (1) is a spline of degree $k$ with the knot points $t_{i}$. In particular, by an appropriate choice of $\tau_{i}$ 's, an arbitrary spline of degree $k$ can be designed on the interval $\left[t_{0}, t_{m}\right]$.

On the other hand, surfaces are generated as

$$
x(s, t)=\sum_{i=-k}^{m_{1}-1} \sum_{j=-k}^{m_{2}-1} \tau_{i, j} B_{k}\left(\alpha\left(s-s_{i}\right)\right) B_{k}\left(\beta\left(t-t_{j}\right)\right) .
$$

where $\alpha, \beta(>0)$ are constants, $m_{1}, m_{2}(>2)$ are integers, and $s_{i}$ 's, $t_{j}$ 's are equally spaced knot points with

$$
s_{i+1}-s_{i}=\frac{1}{\alpha}, \quad t_{i+1}-t_{i}=\frac{1}{\beta} .
$$

This paper is organized as follows: In Section 2, we present normalized uniform B-spline functions. In Section 3 , the problem of designing optimal smoothing curves and surfaces is formulated and optimal solutions are constructed. We analyze the asymptotic and statistical properties of optimal curves and surfaces in Section 4. The results of numerical simulation studies are presented in Section 5, and concluding remarks are given in Section 6.

Here we introduce some of the principal symbols: $\nabla^{2}$ denotes the Laplacian operator, and $\otimes$ the Kronecker product. 'vec' denotes the vec-function, i.e. for a matrix $A=\left[\begin{array}{llll}a_{1} & a_{2} & \cdots & a_{n}\end{array}\right] \in \mathbf{R}^{m \times n}$ with $a_{i} \in \mathbf{R}^{m}$, vec $A=\left[\begin{array}{llll}a_{1}^{T} & a_{2}^{T} & \cdots & a_{n}^{T}\end{array}\right]^{T} \in \mathbf{R}^{m \times n}$ (see e.g. [7]).

\section{Normalized Uniform B-Splines}

The normalized, uniform B-spline function of degree $k$, denoted as $B_{k}(t)$, is defined by

$B_{k}(t)= \begin{cases}N_{k-j, k}(t-j) & j \leq t \leq j+1 \quad j=0, \cdots, k \\ 0 & t \leq 0, k+1 \leq t .\end{cases}$

Here the basis elements $N_{j, k}(t)(j=0,1, \cdots, k)$ are obtained recursively by the following algorithm [14]. Let $N_{0,0}(t) \equiv 1$ and, for $i=1,2, \cdots, k$, compute

$$
\left\{\begin{aligned}
N_{0, i}(t) & =\frac{1-t}{i} N_{0, i-1}(t) \\
N_{j, i}(t) & =\frac{i-j+t}{i} N_{j-1, i-1}(t) \\
& +\frac{1+j-t}{i} N_{j, i-1}(t), j=1, \cdots, i-1 \\
N_{i, i}(t) & =\frac{t}{i} N_{i-1, i-1}(t) .
\end{aligned}\right.
$$

Thus, $B_{k}(t)$ is a piece-wise polynomial of degree $k$ with integer knot points and is $k-1$ times continuously differentiable. It is noted that $B_{k}(t)$ for $k=0,1,2, \cdots$ is normalized in the sense of $\sum_{j=0}^{k} N_{j, k}(t)=1, \quad 0 \leq t \leq$ 1 , and this yields $\int_{-\infty}^{\infty} B_{k}(t) d t=\int_{0}^{k+1} B_{k}(t) d t=1$.
For the sake of later reference, we show here the function $B_{3}(t)$,

$$
B_{3}(t)= \begin{cases}\frac{1}{6} t^{3} & 0 \leq t<1 \\ \frac{1}{6}\left(-3 t^{3}+12 t^{2}-12 t+4\right) & 1 \leq t<2 \\ \frac{1}{6}\left(3 t^{3}-24 t^{2}+60 t-44\right) & 2 \leq t<3 \\ \frac{1}{6}(4-t)^{3} & 3 \leq t<4 \\ 0 & \text { otherwise }\end{cases}
$$

\section{Results on Standard Smoothing Spline Problems}

In this section, we present the basic results on smoothing spline problems. Since cubic splines are used most frequently for practical purposes, we restrict ourselves to the case of $k=3$ in the sequel.

\subsection{Smoothing Spline Curves}

Equation (1) in the case of $k=3$ is written as

$$
x(t)=\sum_{i=-3}^{m-1} \tau_{i} B_{3}\left(\alpha\left(t-t_{i}\right)\right) .
$$

Suppose that we are given a set of data

$$
\mathcal{D}=\left\{\left(u_{i} ; d_{i}\right): u_{i} \in\left[t_{0}, t_{m}\right], d_{i} \in \mathbb{R}, i=1, \cdots, N\right\},
$$

and let $\tau \in \mathbb{R}^{M}(M=m+3)$ be the weight vector defined by

$$
\tau=\left[\begin{array}{llll}
\tau_{-3} & \tau_{-2} & \cdots & \tau_{m-1}
\end{array}\right]^{T} .
$$

Then, a basic problem of optimal smoothing spline curves is to find a curve $x(t)$, or equivalently a vector $\tau \in \mathbf{R}^{M}$, minimizing a cost function,

$$
J(\tau)=\lambda \int_{I}\left(x^{(2)}(t)\right)^{2} d t+\sum_{i=1}^{N} w_{i}\left(x\left(u_{i}\right)-d_{i}\right)^{2},
$$

where $\lambda(>) 0$ is a smoothing parameter, $w_{i}\left(0 \leq w_{i} \leq 1\right)$ are weights for approximation errors, and the integration interval $I$ is taken as either $I=(-\infty,+\infty)$ or $I=\left(t_{0}, t_{m}\right)$.

This problem can be solved as follows. In order to express the right hand side of (11) in terms of $\tau$, we introduce the following notations: Let a vector $b(t) \in$ $\mathbf{R}^{M}$ and a matrix $B \in \mathbf{R}^{M \times N}$ be

$$
\begin{aligned}
b(t)= & {\left[\begin{array}{cc}
B_{3}\left(\alpha\left(t-t_{-3}\right)\right) & B_{3}\left(\alpha\left(t-t_{-2}\right)\right) \\
\cdots & B_{3}\left(\alpha\left(t-t_{m-1}\right)\right)
\end{array}\right]^{T}, } \\
B= & {\left[\begin{array}{llll}
b\left(u_{1}\right) & b\left(u_{2}\right) & \cdots & b\left(u_{N}\right)
\end{array}\right] . }
\end{aligned}
$$

Then, noting that $x(t)$ is expressed as $x(t)=\tau^{T} b(t)$, we can show that the cost function is written as

$$
J(\tau)=\lambda \tau^{T} Q \tau+\left(B^{T} \tau-d\right)^{T} W\left(B^{T} \tau-d\right) .
$$


Here, $Q \in \mathbf{R}^{M \times M}$ is a Gramian defined by

$$
Q=\int_{I} \frac{d^{2} b(t)}{d t^{2}} \frac{d^{2} b^{T}(t)}{d t^{2}} d t
$$

and

$$
\begin{gathered}
W=\operatorname{diag}\left\{w_{1}, w_{2}, \cdots, w_{N}\right\}, \\
d=\left[\begin{array}{llll}
d_{1} & d_{2} & \cdots & d_{N}
\end{array}\right]^{T} .
\end{gathered}
$$

We then see that optimal weight $\tau$ minimizing $J(\tau)$ in (14) is obtained as a solution of

$$
\left(\lambda Q+B W B^{T}\right) \tau=B W d .
$$

Note that this equation has at least one solution, since in general the relation

$$
\operatorname{rank}\left(S+U U^{T}\right)=\operatorname{rank}\left[S+U U^{T}, U v\right]
$$

holds for any matrices $S=S^{T} \geq 0, U$ and vector $v$ of compatible dimensions. Obviously, the solution of (18) is unique if and only if $\lambda Q+B W B^{T}>0$.

By changing the integration variable in (15), the Gramian $Q$ can be transformed as

$$
Q=\alpha^{3} R
$$

Here $R \in \mathbf{R}^{M \times M}$ is a Gramian defined by

$$
R=\int_{\hat{I}} \hat{b}^{(2)}(t)\left(\hat{b}^{(2)}(t)\right)^{T} d t
$$

where $\hat{I}=(-\infty,+\infty)$ if $I=(-\infty,+\infty)$ and $\hat{I}=(0, m)$ if $I=\left(t_{0}, t_{m}\right)$, and

$$
\begin{aligned}
& \hat{b}(t)=\left[\begin{array}{lll}
B_{3}(t-(-3)) & B_{3}(t-(-2)) & \cdots
\end{array}\right. \\
& \left.\cdots \quad B_{3}(t-(m-1))\right]^{T} .
\end{aligned}
$$

Then we can show that the following properties hold.

Lemma 1 The Gramian $Q$ in (15) is nonsingular if $I=(-\infty,+\infty)$, and singular if $I=\left(t_{0}, t_{m}\right)$.

Moreover, using the function $B_{3}(t)$ in (7), we can compute the integral in (21). Denoting the matrix $R$ for $\hat{I}=(-\infty,+\infty)$ by $R_{\infty}$, we obtain

$$
R_{\infty}=\frac{1}{6}\left[\begin{array}{cccccccc}
16 & -9 & 0 & 1 & & & & \\
-9 & 16 & -9 & 0 & 1 & & & \\
0 & -9 & 16 & -9 & 0 & 1 & & \\
1 & 0 & -9 & 16 & -9 & 0 & 1 & \\
& \ddots & & & \ddots & & & \ddots \\
& & \ddots & & & \ddots & & \\
& & & \ddots & & & \ddots & \\
& & & & 1 & 0 & -9 & 16
\end{array}\right],
$$

and $R_{F}$ for $\hat{I}=(0, m)$, is given by

$$
\begin{aligned}
R_{F} & =\left(\int_{-\infty}^{+\infty}-\int_{-\infty}^{0}-\int_{m}^{+\infty}\right) \hat{b}^{(2)}(t)\left(\hat{b}^{(2)}(t)\right)^{T} d t \\
& =R_{\infty}-\left(R_{-}+R_{+}\right),
\end{aligned}
$$

where the second and third terms can be computed explicitly as

$$
\begin{aligned}
& R_{-}=\frac{1}{6}\left[\begin{array}{ccc|c}
14 & -6 & 0 & \\
-6 & 8 & -3 & 0_{3, M-3} \\
0 & -3 & 2 & \\
\hline 0_{M-3,3} & 0_{M-3, M-3}
\end{array}\right], \\
& R_{+}=\frac{1}{6}\left[\begin{array}{c|ccc}
0_{M-3, M-3} & \multicolumn{3}{|c}{0_{M-3,3}} \\
\hline 0_{3, M-3} & -3 & -3 & 0 \\
& 0 & -6 & 14
\end{array}\right] .
\end{aligned}
$$

Remark 1 If $I=(-\infty,+\infty)$, then $\lambda Q+B W B^{T}$ is nonsingular by Lemma 1, and thus (18) has a unique solution. On the other hand, when $I=\left(t_{0}, t_{m}\right)$, the matrix $\lambda Q+B W B^{T}$ may be singular. In such a case, we employ the so-called minimum norm solution for (18), which is guaranteed to be unique.

\subsection{Smoothing Spline Surfaces}

Equation (3) in the case of $k=3$ is written as

$$
x(s, t)=\sum_{i=-3}^{m_{1}-1} \sum_{j=-3}^{m_{2}-1} \tau_{i, j} B_{3}\left(\alpha\left(s-s_{i}\right)\right) B_{3}\left(\beta\left(t-t_{j}\right)\right) .
$$

Then, choosing appropriate weighting coefficient $\tau_{i, j}$ called 'control point', $\cdot x(s, t)$ can represent an arbitrary spline surface on the rectangular domain $\mathcal{S}=$ $\left[s_{0}, s_{m_{1}}\right] \times\left[t_{0}, t_{m_{2}}\right] \subset \mathbf{R}^{2}$. Now suppose that a set of spatial data

$$
\begin{array}{r}
\mathcal{D}=\left\{\left(u_{i}, v_{j} ; d_{i j}\right):\left(u_{i}, v_{j}\right) \in \mathcal{S}, d_{i j} \in \mathbf{R},\right. \\
\left.i=1,2, \cdots, N_{1}, j=1,2, \cdots, N_{2}\right\}
\end{array}
$$

is given. Letting $\tau=\left[\tau_{i, j}\right] \in \mathbb{R}^{M_{1} \times M_{2}}$ be the weight matrix with $M_{1}=m_{1}+3$ and $M_{2}=m_{2}+3$, we consider the following cost function for a design of the smoothing surface:

$$
\begin{aligned}
J(\tau)= & \lambda \int_{I_{1}} \int_{I_{2}}\left(\nabla^{2} x(s, t)\right)^{2} d s d t \\
& +\sum_{i=1}^{N_{1}} \sum_{j=1}^{N_{2}} w_{i j}\left(x\left(u_{i}, v_{j}\right)-d_{i j}\right)^{2},
\end{aligned}
$$

where $I_{1}, I_{2}$ are some intervals in $(-\infty,+\infty), \lambda(>0)$ is a smoothing parameter, and $w_{i j}\left(0 \leq w_{i j} \leq 1\right)$ denotes weights for approximation errors.

Minimization of this cost function proceeds as in the previous case. In order to express the right hand side of (28) in terms of $\tau$, we introduce the following notations. Let $b_{1}(t) \in \mathbb{R}^{M_{1}}$ and $b_{2}(t) \in \mathbb{R}^{M_{2}}$ be

$$
\begin{aligned}
& b_{1}(s)=\left[B_{3}\left(\alpha\left(s-s_{-3}\right)\right) B_{3}\left(\alpha\left(s-s_{-2}\right)\right)\right. \\
& \left.\cdots B_{3}\left(\alpha\left(s-s_{m_{1}-1}\right)\right)\right]^{T} \\
& b_{2}(t)=\left[B_{3}\left(\beta\left(t-t_{-3}\right)\right) B_{3}\left(\beta\left(t-t_{-2}\right)\right)\right. \\
& \left.\cdots B_{3}\left(\beta\left(t-t_{m_{2}-1}\right)\right)\right]^{T} \text {. }
\end{aligned}
$$


Then with $\hat{\tau}=\operatorname{vec} \tau\left(\in \mathbf{R}^{M_{1} M_{2}}\right), x(s, t)$ in (27) can be written as

$$
x(s, t)=\left(b_{2}(t) \otimes b_{1}(s)\right)^{T} \hat{\tau},
$$

and the cost function in (28) is obtained in terms of $\hat{\tau}$ as

$$
\begin{aligned}
& J(\hat{\tau})=\lambda \hat{\tau}^{T} Q \hat{\tau} \\
& +\left(\left(B_{2} \otimes B_{1}\right)^{T} \hat{\tau}-d\right)^{T} W\left(\left(B_{2} \otimes B_{1}\right)^{T} \hat{\tau}-d\right)
\end{aligned}
$$

Here, $Q \in \mathbf{R}^{M_{1} M_{2} \times M_{1} M_{2}}$ is a Gramian defined by

$$
\begin{aligned}
Q= & Q_{2}^{(00)} \otimes Q_{1}^{(22)}+Q_{2}^{(02)} \otimes\left(Q_{1}^{(02)}\right)^{T} \\
& +\left(Q_{2}^{(02)}\right)^{T} \otimes Q_{1}^{(02)}+Q_{2}^{(22)} \otimes Q_{1}^{(00)}
\end{aligned}
$$

where $Q_{l}^{(i j)} \in \mathbf{R}^{M_{l} \times M_{l}}(l=1,2 ; i, j=0,1,2)$ are given by

$$
Q_{l}^{(i j)}=\int_{I_{l}} \frac{d^{i} b_{l}(t)}{d t^{i}} \frac{d^{j} b_{l}^{T}(t)}{d t^{j}} d t .
$$

Note that $Q=Q^{T} \geq 0$ since

$$
\hat{\tau}^{T} Q \hat{\tau}=\int_{I_{1}} \int_{I_{2}}\left(\nabla^{2} x(s, t)\right)^{2} d s d t \geq 0 \quad \forall \hat{\tau} .
$$

Moreover, in (32), matrices $B \in \mathbf{R}^{M_{1} \times N_{1}}$ and $B \in$ $\mathrm{R}^{M_{2} \times N_{2}}$ are defined by

$$
\begin{aligned}
& B_{1}=\left[\begin{array}{llll}
b_{1}\left(u_{1}\right) & b_{1}\left(u_{2}\right) & \cdots & b_{1}\left(u_{N_{1}}\right)
\end{array}\right], \\
& B_{2}=\left[\begin{array}{llll}
b_{2}\left(v_{1}\right) & b_{2}\left(v_{2}\right) & \cdots & b_{2}\left(v_{N_{2}}\right)
\end{array}\right],
\end{aligned}
$$

and $W \in \mathbf{R}^{N_{1} N_{2} \times N_{1} N_{2}}$ and $d \in \mathbf{R}^{N_{1} N_{2}}$ by

$$
\begin{array}{r}
W=\quad \operatorname{diag}\left\{w_{11}, w_{21}, \cdots, w_{N_{1} 1}, \cdots,\right. \\
\left.w_{1 N_{2}}, w_{2 N_{2}}, \cdots, w_{N_{1} N_{2}}\right\} \\
d=\left[\begin{array}{l}
d_{11}, \\
d_{21}, \cdots, d_{N_{1} 1}, \cdots, \\
\left.d_{1 N_{2}}, d_{2 N_{2}}, \cdots, d_{N_{1} N_{2}}\right]^{T} .
\end{array}\right.
\end{array}
$$

Then, the optimal weight $\hat{\tau}$ minimizing the cost function in (32) is obtained as a solution of

$$
\left(\lambda Q+\left(B_{2} \otimes B_{1}\right) W\left(B_{2} \otimes B_{1}\right)^{T}\right) \hat{\tau}=\left(B_{2} \otimes B_{1}\right) W d .
$$

Note that, by (19), this equation is consistent and always has at least one solution.

For the sake of analyses and numerical computations of $Q$ matrix, it is convenient to introduce the following matrices $R_{l}^{(i j)} \in \mathbf{R}^{M_{l} \times M_{l}}(l=1,2 ; i, j=0,1,2)$,

$$
R_{l}^{(i j)}=\int_{\hat{I}_{l}} \hat{b}_{l}^{(i)}(t)\left(\hat{b}_{l}^{(j)}(t)\right)^{T} d t
$$

where $\hat{I}_{l}=(-\infty,+\infty)$ if $I_{l}=(-\infty,+\infty)$ for $l=1,2$, and $\hat{I}_{1}=\left(0, m_{1}\right), \hat{I}_{2}=\left(0, m_{2}\right)$ if $I_{1}=\left(s_{0}, s_{m_{1}}\right), I_{2}=$ $\left(t_{0}, t_{m_{2}}\right)$. Moreover the vector $\hat{b}_{l}(t) \in \mathbb{R}^{M_{l}}$ is defined by

$$
\begin{aligned}
& \hat{b}_{l}(t)=\left[\begin{array}{lll}
B_{3}(t-(-3)) & B_{3}(t-(-2)) & \ldots
\end{array}\right. \\
& \left.\cdots B_{3}\left(t-\left(m_{l}-1\right)\right)\right]^{T} .
\end{aligned}
$$

Note that the matrices $R_{l}^{(i j)}$ in (38) are obtained by changing the integration variable in (34) and are related to $Q_{l}^{(i j)}$ as

$$
Q_{1}^{(i j)}=\alpha^{i+j-1} R_{1}^{(i j)}, \quad Q_{2}^{(i j)}=\beta^{i+j-1} R_{2}^{(i j)} .
$$

Regarding the matrix $Q$ in (33), the following properties hold.

Lemma 2 The matrix $Q$ in (33) is nonsingular if $I_{1}=$ $I_{2}=(-\infty,+\infty)$, and singular if $I_{1}=\left(s_{0}, s_{m_{1}}\right)$ and $I_{2}=\left(t_{0}, t_{m_{2}}\right)$.

The matrix $Q$ can be formed easily as in the case of Section 3.1, and is shown in Appendix A. Moreover, by Lemma 2, there always exists a unique solution of (37) when $I_{1}=I_{2}=(-\infty,+\infty)$, whereas such a uniqueness is not guaranteed in the case of $I_{1}=\left(s_{0}, s_{m_{1}}\right)$ and $I_{2}=$ $\left(t_{0}, t_{m_{2}}\right)$

\section{Smoothing Spline Curves for Sampled Data}

In this section, we analyze various properties of smoothing spline curves derived in Section 3.1. In particular, we analyze asymptotic and statistical properties assuming that the data is obtained by sampling some curve with or without noises.

Let the data $d_{i}$ in (9) be obtained by sampling a curve $f(t)$ which is assumed to be continuous in the interval $\left[t_{0}, t_{m}\right]$. In order to analyze asymptotic properties of spline curves as the number of data points $N$ increases, we consider the following cost function instead of (11),

$$
J_{N}(\tau)=\lambda \int_{I}\left(x^{(2)}(t)\right)^{2} d t+\frac{1}{N} \sum_{i=1}^{N}\left(x\left(u_{i}\right)-f\left(u_{i}\right)\right)^{2} .
$$

When the data $d_{i}$ is obtained by sampling the function $f(t)$ with additive noises

$$
d_{i}=f\left(u_{i}\right)+\epsilon_{i}, i=1,2, \cdots, N,
$$

we consider the following cost function

$$
J_{N}^{\epsilon}(\tau)=\lambda \int_{I}\left(x^{(2)}(t)\right)^{2} d t+\frac{1}{N} \sum_{i=1}^{N}\left(x\left(u_{i}\right)-f\left(u_{i}\right)-\epsilon_{i}\right)^{2} .
$$

We assume that the noises are zero-mean and white, namely $\mathrm{E}\left\{\epsilon_{i}\right\}=0$ and $\mathrm{E}\left\{\epsilon_{i} \epsilon_{j}\right\}=\sigma^{2} \delta_{i j}$ for all $i, j$. Moreover, in order to analyze the asymptotic properties, we introduce the cost function:

$J_{c}(\tau)=\lambda \int_{I}\left(x^{(2)}(t)\right)^{2} d t+\int_{t_{0}}^{t_{m}}(x(t)-f(t))^{2} d t$.

The solutions that minimize the cost functions $J_{N}(\tau)$, $J_{N}^{\epsilon}(\tau)$ and $J_{c}(\tau)$ are obtained as follows. The first two cases follow directly from the results in Section 3.1: The 
solution $\tau_{N}$ minimizing $J_{N}(\tau)$ is obtained as a solution of

$$
\left(\lambda Q+\frac{1}{N} B B^{T}\right) \tau_{N}=\frac{1}{N} B f
$$

where $Q$ and $B$ are given in (15) and (13) respectively, and $f=\left[\begin{array}{llll}f\left(u_{1}\right) & f\left(u_{2}\right) & \cdots & f\left(u_{N}\right)\end{array}\right]^{T}$. Obviously, $\tau_{N}^{\epsilon}$ minimizing $J_{N}^{\epsilon}(\tau)$ is a solution of

$$
\left(\lambda Q+\frac{1}{N} B B^{T}\right) \tau_{N}^{\epsilon}=\frac{1}{N} B(f+\epsilon),
$$

where $\epsilon=\left[\begin{array}{llll}\epsilon_{1} & \epsilon_{2} & \cdots & \epsilon_{N}\end{array}\right]^{T}$. On the other hand, $J_{c}(\tau)$ can be written as

$$
\begin{aligned}
& J_{c}(\tau)=\tau^{T}\left(\lambda Q+Q^{(0)}\right) \tau \\
&-2 \tau^{T} \int_{t_{0}}^{t_{m}} b(t) f(t) d t+\int_{t_{0}}^{t_{m}} f^{2}(t) d t
\end{aligned}
$$

where

$$
Q^{(0)}=\int_{t_{0}}^{t_{m}} b(t) b^{T}(t) d t
$$

Thus optimal $\tau$ denoted by $\tau_{c}$ is obtained as a solution of

$$
\left(\lambda Q+Q^{(0)}\right) \tau_{c}=\int_{t_{0}}^{t_{m}} b(t) f(t) d t .
$$

Here we can show that $Q^{(0)}=\left(Q^{(0)}\right)^{T}>0$, hence optimal $\tau_{c}$ exists uniquely. Moreover, $Q^{(0)}$ can be obtained explicitly as in the case of $R_{F}$ in (24).

Convergence properties are established under the following assumption.

(A1) The sample points $u_{i}, i=1,2, \cdots, N$, are such that

$$
\lim _{N \rightarrow \infty} \frac{1}{N} \sum_{i=1}^{N} g\left(u_{i}\right)=\int_{t_{0}}^{t_{m}} g(t) d t
$$

for every continuous function $g(t)$ in $\left[t_{0}, t_{m}\right]$.

We now have the following results for the case $I=$ $(-\infty,+\infty)$.

Theorem 1 Assume that the integration interval is $I=(-\infty,+\infty)$ and that the condition (A1) holds. Then,

(i) The optimal solutions $\tau_{N}, \tau_{N}^{\epsilon}$ and $\tau_{c}$ exist uniquely.

(ii) $\tau_{N}$ converges to $\tau_{c}$ as $N \rightarrow \infty$.

(iii) $\mathrm{E}\left\{\tau_{N}^{\epsilon}\right\}=\tau_{N}$ and $\tau_{N}^{\epsilon}$ converges to $\tau_{c}$ as $N \rightarrow \infty$ in the mean squares sense.

On the other hand, when $I=\left(t_{0}, t_{m}\right)$, the matrix $Q$ is singular. Thus it is possible that the coefficient matrix $\lambda Q+\frac{1}{N} B B^{T}$ in (45) and (46) becomes singular depending on the matrix $B$, i.e. on the data points $u_{i}, i=1, \cdots, N$. In such a case, we employ the minimum norm solution as before, and the following corollary holds.
Corollary 1 Assume that integration interval $I$ is $I=$ $\left(t_{0}, t_{m}\right)$ and that the condition (A1) holds. Then, the same assertions hold as in Theorem 1 with the understanding that we employ the minimum norm solutions for equation (45) and (46).

\section{Smoothing Spline Surfaces for Sampled Data}

We assume that the data $d_{i j}$ in (28) for constructing smoothing surfaces is obtained by sampling a function $f(s, t)$ which is assumed to be continuous in both variables in $\left[s_{0}, s_{m_{1}}\right] \times\left[t_{0}, t_{m_{2}}\right]$. Corresponding to the cost function $J(\tau)$ in (28), we consider the following cost functions,

$$
\begin{array}{r}
J_{N_{1}, N_{2}}(\tau)=\lambda \int_{I_{1}} \int_{I_{2}}\left(\nabla^{2} x(s, t)\right)^{2} d s d t \\
+\frac{1}{N_{1} N_{2}} \sum_{i=1}^{N_{1}} \sum_{j=1}^{N_{2}}\left(x\left(u_{i}, v_{j}\right)-f\left(u_{i}, v_{j}\right)\right)^{2} \\
J_{N_{1}, N_{2}}^{\epsilon}(\tau)=\lambda \int_{I_{1}} \int_{I_{2}}\left(\nabla^{2} x(s, t)\right)^{2} d s d t \\
+\frac{1}{N_{1} N_{2}} \sum_{i=1}^{N_{1}} \sum_{j=1}^{N_{2}}\left(x\left(u_{i}, v_{j}\right)-f\left(u_{i}, v_{j}\right)-\epsilon_{i j}\right)^{2} .
\end{array}
$$

In (52), the noises $\epsilon_{i j}$ are assumed to be zero-mean and white, i.e. $\mathrm{E}\left\{\epsilon_{i j}\right\}=0 \forall i, j, \mathrm{E}\left\{\epsilon_{i j} \epsilon_{k l}\right\}=\sigma^{2}$ for $i=$ $j=k=l$, and $\mathrm{E}\left\{\epsilon_{i j} \epsilon_{k l}\right\}=0$ otherwise. Moreover, in order to analyze convergence properties of solutions as $N_{1}, N_{2} \rightarrow \infty$, we introduce the following cost function.

$$
\begin{aligned}
J_{c}(\tau) & =\lambda \int_{I_{1}} \int_{I_{2}}\left(\nabla^{2} x(s, t)\right)^{2} d s d t \\
+ & \int_{s_{0}}^{s_{m}} \int_{t_{0}}^{t_{n}}(x(s, t)-f(s, t))^{2} d s d t .
\end{aligned}
$$

Using the results established in Section 3.2, we easily see that the optimal solutions $\tau_{N_{1}, N_{2}}$ and $\tau_{N_{1}, N_{2}}^{\epsilon}$ minimizing the cost functions in (51) and (52) are obtained respectively as solutions of

$$
\mathcal{A}_{N_{1}, N_{2}} \hat{\tau}_{N_{1}, N_{2}}=\frac{1}{N_{1} N_{2}}\left(B_{2} \otimes B_{1}\right) f
$$

and

$$
\mathcal{A}_{N_{1}, N_{2}} \hat{\tau}_{N_{1}, N_{2}}^{\epsilon}=\frac{1}{N_{1} N_{2}}\left(B_{2} \otimes B_{1}\right)(f+\epsilon),
$$

where $\mathcal{A}_{N_{1}, N_{2}} \in \mathbf{R}^{M_{1} M_{2} \times M_{1} M_{2}}$ is given by

$$
\mathcal{A}_{N_{1}, N_{2}}=\lambda Q+\frac{1}{N_{1} N_{2}}\left(B_{2} \otimes B_{1}\right)\left(B_{2} \otimes B_{1}\right)^{T},
$$

and the vectors $f \in \mathbf{R}^{N_{1} N_{2}}$ and $\epsilon \in \mathbf{R}^{N_{1} N_{2}}$ are defined by

$$
\begin{aligned}
f= & {\left[f\left(u_{1}, v_{1}\right), f\left(u_{2}, v_{1}\right), \cdots, f\left(u_{N_{1}}, v_{1}\right), \cdots,\right.} \\
& \left.f\left(u_{1}, v_{N_{2}}\right), f\left(u_{2}, v_{N_{2}}\right), \cdots, f\left(u_{N_{1}}, v_{N_{2}}\right)\right]^{T}, \\
\epsilon=\quad & {\left[\epsilon_{11}, \epsilon_{21}, \cdots, \epsilon_{N_{1} 1}, \cdots,\right.} \\
& \left.\epsilon_{1 N_{2}}, \epsilon_{2 N_{2}}, \cdots, \epsilon_{N_{1} N_{2}}\right]^{T} .
\end{aligned}
$$


On the other hand, we can show that the optimal solution for the cost function in (53) is obtained as the solution of

$$
\left(\lambda Q+R_{2}^{(00)} \otimes R_{1}^{(00)}\right) \hat{\tau}_{c}=\phi,
$$

where $\phi \in \mathbf{R}^{M_{1} M_{2}}$ is defined by

$$
\phi=\int_{I_{1}} \int_{I_{2}} f(s, t)\left(b_{2}(t) \otimes b_{1}(s)\right) d s d t .
$$

Now we introduce the following assumption:

(A2) The sample points $\left(u_{i}, v_{j}\right), i=1, \cdots, N_{1}, j=$ $1, \cdots, N_{2}$, are such that

$$
\lim _{N_{1}, N_{2} \rightarrow \infty} \frac{1}{N_{1} N_{2}} \sum_{i=1}^{N_{1}} \sum_{j=1}^{N_{2}} g\left(u_{i}, v_{j}\right)=\int_{s_{0}}^{s_{m_{1}}} \int_{t_{0}}^{t_{m_{2}}} g(s, t) d s d t
$$

for every continuous function $g(s, t)$ in $\left[s_{0}, s_{m_{1}}\right] \times$ $\left[t_{0}, t_{m_{2}}\right]$.

Then we obtain the following result.

Theorem 2 Assume that integration intervals $I_{1}, I_{2}$ are $I_{1}=I_{2}=(-\infty,+\infty)$ and that the condition (A2) holds. Then,

(i) The optimal solutions $\tau_{N_{1}, N_{2}}, \tau_{N_{1}, N_{2}}^{\epsilon}$ and $\tau_{c}$ exist uniquely.

(ii) $\tau_{N_{1}, N_{2}}$ converges to $\tau_{c}$ as $N_{1}, N_{2} \rightarrow \infty$.

(iii) $\mathrm{E}\left\{\tau_{N_{1}, N_{2}}^{\epsilon}\right\}=\tau_{N_{1}, N_{2}}$ and $\tau_{N_{1}, N_{2}}^{\epsilon}$ converges to $\tau_{c}$ as $N_{1}, N_{2} \rightarrow \infty$ in the mean squares sense.

In the case where the integration intervals are $I_{1}=$ $\left(s_{0}, s_{m_{1}}\right)$ and $I_{1}=\left(t_{0}, t_{m_{2}}\right)$, Lemma 2 shows that the coefficient matrix $\mathcal{A}_{N_{1}, N_{2}}$ in (54) and (55) may be singular since the matrix $Q$ is singular, in which case we use the minimum norm solution. Note that (57) always has a unique solution since it holds that the matrix $R_{2}^{(00)} \otimes R_{1}^{(00)}$ is positive-definite. Thus we have the following corollary.

Corollary 2 Assume that the integration intervals $I_{1}, I_{2}$ are $I_{1}=\left(s_{0}, s_{m_{1}}\right)$ and $I_{2}=\left(t_{0}, t_{m_{2}}\right)$ and that the condition (A2) holds. Then, the same assertions hold as in Theorem 2 with the understanding that we employ the minimum norm solutions for equations (54) and (55).

\section{Simulation Studies}

We construct optimal smoothing curves and surfaces for the case $k=3$. The discrete data are generated by sampling the following function.

$f(s, t)=2+5 \exp \{-|(s-5)(t-5)| / 10\}+\sin (s) \cos (2 t)$.
First we consider the case of curves where we set $\alpha=1$ and $m=10, t_{0}=0, t_{m}=10$. We generated 10 noisy discrete data (i.e. $N=10$ ) using the function $f(2, t)$ (i.e. we fix $s=2$ in $f(s, t))$ at equallyspaced sampling points with $\sigma=0.1$. The optimal spline curves $x_{c}(t)$ and $x_{N}^{\epsilon}(t)$ are shown in Figure 1 together with the original curve $f(2, t)$. We see that the curve $x_{c}(t)$, i.e. $\lim _{N \rightarrow \infty} x_{N}(t)$, approximates closely the original curve.

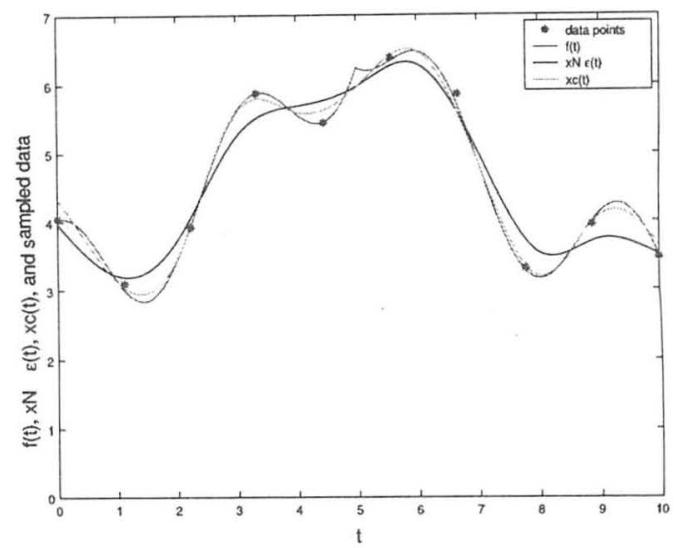

Fig. 1: The curves $f(t)$ (red line), $x_{c}(t)$ (green line) and $x_{N}^{\epsilon}(t)$ (balck line) with $N=10$ and $\sigma=0.1$.

Next we construct optimal smoothing surfaces for the case $\alpha=\beta=1, s_{0}=t_{0}=0, m=n=10$ and thus $s_{m}=t_{n}=10$. The surface data is generated by sampling the function $f(s, t)$ with the noise magnitude $\sigma=0.5$. As we see from Figure $2, x_{N_{1} N_{2}}^{\epsilon}(s, t)$ approximates the original surface $f(s, t)$ more accurately as the number of data $N_{1}, N_{2}$ increases, and eventually converges to $x_{c}(s, t)$.

\section{Concluding Remarks}

We considered the problem of designing optimal smoothing spline curves and surfaces for: a given set of discrete data. By employing normalized uniform Bsplines as the basis functions, we derived concise expressions for the optimal curves and surfaces, and their numerical computation procedures are straightforward. Then, assuming that a set of data in a plane is obtained by sampling some continuous curve with or without noises, we proved that, under a very natural condition, optimal smoothing splines converge to some limiting curve as the number of data increases. The cases of surfaces are treated in parallel, and we see that the extensions of the results for one-dimensional case (i.e. curves) to the two-dimensional case (i.e. surfaces) are straightforward. This indicates that the same line of approach can be used for extensions to still higher dimensions. 

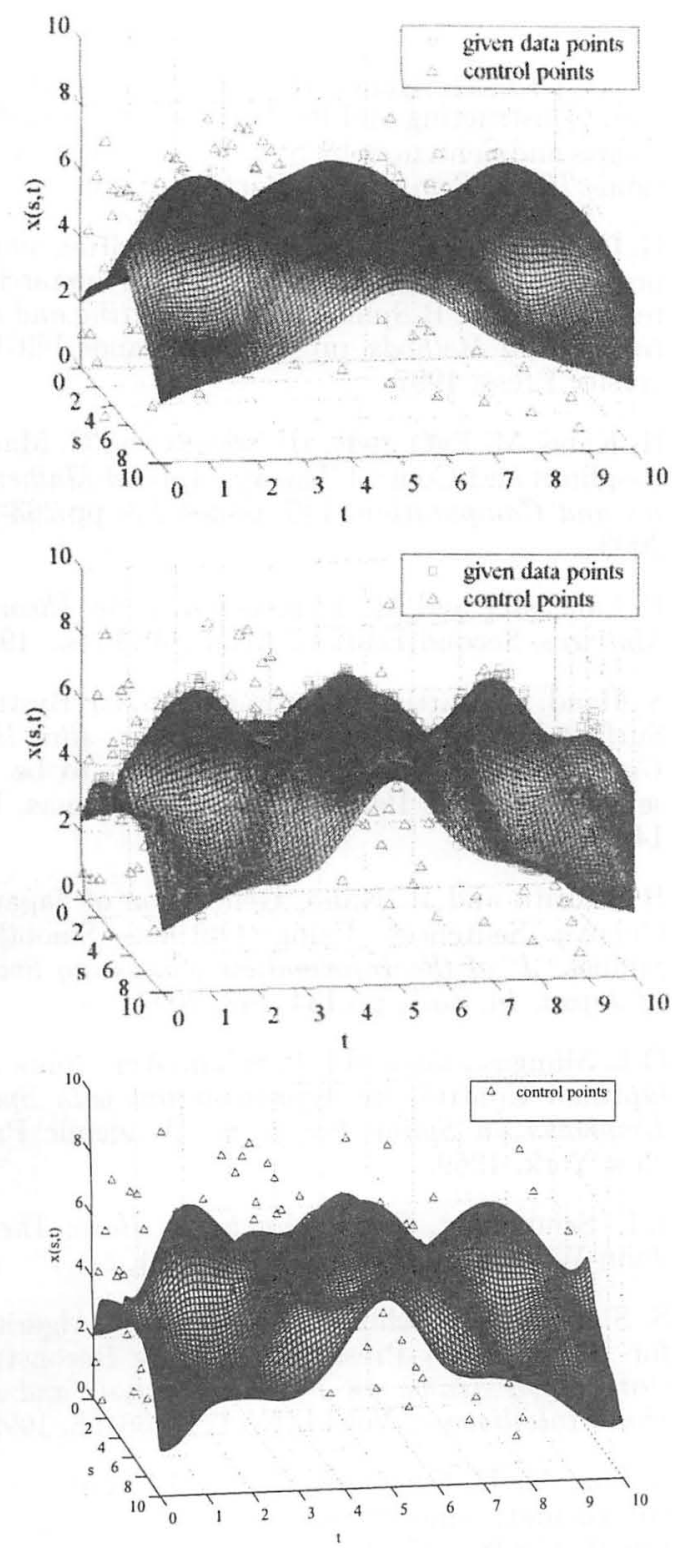

Fig. 2: Optimal smoothing surfaces $x_{N_{1}, N_{2}}^{\epsilon}(s, t)$ with $N_{1}=N_{2}=10$ (top) and $N_{1}=N_{2}=40$ (middle), and $x_{c}(s, t)$ (bottom).

\section{A Computing Matrix $Q$ in Section 3.2}

By (40), it suffices to compute the matrices $R_{l}^{(i j)}$ in (38) for constructing the matrix $Q$ in (33). As we will see, these matrices are of very special structure and can be readily set up using precomputed values.

\section{A.1 The case where $I_{l}=(-\infty,+\infty)$}

We first note that integrating (38) by parts yields $R_{l}^{(02)}=-R_{l}^{(11)}$ and hence it suffices to derive expressions for $R_{l}^{(i i)}(i=0,1,2)$ to obtain $Q$. Noting that the elements in matrices $R_{1}^{(i i)}$ and $R_{2}^{(i i)}$ are similarly defined, we can denote the elements of $R_{l}^{(i i)}$ as

$$
R_{l}^{(i i)}=\left[r_{p, q}^{(i i)}\right]_{p, q=-3}^{m_{l}-1}
$$

Procedure 1 (i) Set up the matrices $R_{l}^{(i i)} \in$ $\mathbf{R}^{M_{l} \times M_{l}}(l=1,2 ; i=0,1,2)$ using Table 1.

(ii) Let $R_{l}^{(02)}=-R_{l}^{(11)}$ for $l=1,2$.

(iii) Compute $Q$ by (37) and (40).

Note that, from this table, we obtain the matrix $R_{l}^{(22)}=\left[r_{p, q}^{(22)}\right]_{p, q=-3}^{m_{l}-1}$, which is of the form (23).

Table 1: The elements $r_{p q}^{(i i)}$ of matrix $R_{l}^{(i i)}(l=1,2$ and $i=0,1,2)$

\begin{tabular}{|c|c|c|c|}
\hline & $r_{p q}^{(00)}$ & $r_{p q}^{(11)}$ & $r_{p q}^{(22)}$ \\
\hline \hline$p-q=0$ & $\frac{151}{315}$ & $\frac{2}{3}$ & $\frac{8}{3}$ \\
\hline$|p-q|=1$ & $\frac{397}{1680}$ & $-\frac{1}{8}$ & $-\frac{3}{2}$ \\
\hline$|p-q|=2$ & $\frac{1}{42}$ & $-\frac{1}{5}$ & 0 \\
\hline$|p-q|=3$ & $\frac{1}{5040}$ & $-\frac{1}{12}$ & $\frac{1}{6}$ \\
\hline otherwise & 0 & 0 & 0 \\
\hline
\end{tabular}

A.2 The case where $I_{1}=\left(s_{0}, s_{m_{1}}\right)$ and $I_{2}=$ $\left(t_{0}, t_{m_{2}}\right)$

We first rewrite $R_{l}^{(i j)} \in \mathbf{R}^{M_{l} \times M_{l}}$ as

$$
R_{l}^{(i j)}=\left(\int_{-\infty}^{+\infty}-\int_{-\infty}^{0}-\int_{m_{l}}^{+\infty}\right) \hat{b}_{l}^{(i)}(t)\left(\hat{b}_{l}^{(j)}(t)\right)^{T} d t,
$$

and these three terms as

$$
R_{l}^{(i j)}={ }^{\infty} R_{l}^{(i j)}-U_{l}^{(i j)}-V_{l}^{(i j)} .
$$

Here note that the first term ${ }^{\infty} R_{l}^{(i j)}$ was computed in Section A.1. Also let

$$
U_{l}^{(02)}=U_{l}^{c}-U_{l}^{(11)}, \quad V_{l}^{(02)}=V_{l}^{c}-V_{l}^{(11)},
$$

where $U_{l}^{c}$ and $V_{l}^{c}$ are given by

$$
\begin{aligned}
& U_{l}^{c}=\frac{1}{12}\left[\begin{array}{ccc|c}
-1 & 0 & 1 & \\
-4 & 0 & 4 & 0_{3, M_{l}-3} \\
-1 & 0 & 1 & \\
\hline 0_{M_{l}-3,3} & 0_{M_{l}-3, M_{l}-3}
\end{array}\right], \\
& V_{l}^{c}=\frac{1}{12}\left[\begin{array}{c|ccc}
0_{M_{l}-3, M_{l}-3} & \multicolumn{3}{|c}{0_{M_{l}-3,3}} \\
\hline 0_{3, M_{l}-3} & 1 & 0 & -1 \\
& 1 & 0 & -4 \\
& 1 & 0 & -1
\end{array}\right] .
\end{aligned}
$$

Procedure 2 (i) Set up the matrices $U_{l}^{(i i)}, V_{l}^{(i i)}$ for $l=1,2 ; i=0,1,2$ using Tables 2 and 3 respectively.

(ii) Compute $R_{l}^{(i i)}(l=1,2 ; i=0,1,2)$ by (59). 
(iii) Compute $U_{l}^{(02)}, V_{l}^{(02)}(l=1,2)$ by (60), (61), (62), and let $U_{l}^{(20)}=\left(U_{l}^{(02)}\right)^{T}$ and $V_{l}^{(20)}=$ $\left(V_{l}^{(02)}\right)^{T}$

(iv) Compute $R_{l}^{(02)}(l=1,2)$ by (59) and let $R_{l}^{(20)}=$ $\left(R_{l}^{(02)}\right)^{T} \quad(l=1,2)$.

(v) Finally, compute $Q$ by (37) and (40).

Note that, by this procedure, we obtain the matrices $U_{l}^{(22)}$ and $V_{l}^{(22)}$ in the form of (25) and (26) respectively.

Table 2: The elements ${ }^{l} u_{p q}^{(i i)}$ of matrix $U_{l}^{(i i)}(l=1,2$ and $i=0,1,2)$

\begin{tabular}{|c|c|c|c|}
\hline$(p, q)$ & ${ }^{l} u_{p q}^{(00)}$ & ${ }^{l} u_{p q}^{(11)}$ & ${ }^{l} u_{p q}^{(22)}$ \\
\hline \hline$(-3,-3)$ & $\frac{599}{1260}$ & $\frac{37}{60}$ & $\frac{7}{3}$ \\
\hline$(-2,-2)$ & $\frac{151}{630}$ & $\frac{1}{3}$ & $\frac{4}{3}$ \\
\hline$(-1,-1)$ & $\frac{1}{252}$ & $\frac{1}{20}$ & $\frac{1}{3}$ \\
\hline$(-3,-2)$ & $\frac{59}{280}$ & $-\frac{11}{60}$ & -1 \\
\hline$(-2,-1)$ & $\frac{43}{1680}$ & $\frac{7}{120}$ & $-\frac{1}{2}$ \\
\hline$(-3,-1)$ & $\frac{1}{84}$ & $-\frac{1}{10}$ & 0 \\
\hline otherwise & 0 & 0 & 0 \\
\hline
\end{tabular}

Table 3: The elements ${ }^{l} v_{p q}^{(i i)}$ of matrix $V_{l}^{(i i)}(l=1,2$ and $i=0,1,2$ )

\begin{tabular}{|c|c|c|c|}
\hline$(p, q)$ & ${ }^{l} v_{p q}^{(00)}$ & ${ }^{l} v_{p q}^{(11)}$ & ${ }^{l} v_{p q}^{(22)}$ \\
\hline \hline$\left(m_{l}-3, m_{l}-3\right)$ & $\frac{1}{252}$ & $\frac{1}{20}$ & $\frac{1}{3}$ \\
\hline$\left(m_{l}-2, m_{l}-2\right)$ & $\frac{151}{630}$ & $\frac{1}{3}$ & $\frac{4}{3}$ \\
\hline$\left(m_{l}-1, m_{l}-1\right)$ & $\frac{599}{1260}$ & $\frac{37}{60}$ & $\frac{7}{3}$ \\
\hline$\left(m_{l}-3, m_{l}-2\right)$ & $\frac{43}{1680}$ & $\frac{7}{120}$ & $-\frac{1}{2}$ \\
\hline$\left(m_{l}-2, m_{l}-1\right)$ & $\frac{59}{280}$ & $-\frac{11}{60}$ & -1 \\
\hline$\left(m_{l}-3, m_{l}-1\right)$ & $\frac{1}{84}$ & $-\frac{1}{10}$ & 0 \\
\hline otherwise & 0 & 0 & 0 \\
\hline
\end{tabular}

\section{References}

[1] C. de Boor, A practical guide to splines, SpringerVerlag, New York, 1978.

[2] M. Egerstedt and C. Martin, Optimal trajectory planning and smoothing splines, Automatica, 37, pp.1057-1064, 2001.

[3] M. Egerstedt and C. F. Martin: Statistical Estimates for Generalized Splines, Control, Optimisation and Calculus of Variations, Vol.9, pp.553-562, 2003.
[4] H. Fujioka, H. Kano, H. Nakata and H. Shinoda, Constructing and Reconstructing Characters, Words and Sentences by Synthesizing Writing Motions, IEEE Trans. SMC Part A, to appear.

[5] G. Greiner and K. Hormann, Interpolating and approximating scattered 3D data with hierarchical tensor product B-Splines, Surface Fitting and Multiresolution Methods, pp.163-172, Vanderbilt University Press, 1997.

[6] H. Kano, M. Egerstedt, H. Nakata and C. Martin: B-splines and Control Theory, Applied Mathematics and Computation, 145, issues 2-3, pp.263-288, 2003.

[7] P. Lancaster and M. Tismenetsky, The Theory of Matrices, Second Edition, Academic Press, 1985.

[8] S. Maad, C. Martin, T. Strömberg and J. Byström, Surface Fitting with Boundary Data, 43rd IEEE Conference on Decision and Control, to be presented, Atlantis, Paradise Island, Bahamas, Dec. 14-17, 2004.

[9] H. Nakata and H. Kano, Generation of Japanese Cursive Sentences Using Optimal Smoothing Splines, J. of the Information Processing Society of Japan, 44, no.1, pp.134-142, 2003.

[10] O.L. Mangasarian and L.L. Schumaker, Splines via Optimal Control, in Approximation with Special Emphasis on Spline Functions, Academic Press, New York, 1969.

[11] L.L. Schumaker, Spline Functions: Basic Theory, John Wiley \& Sons, New York, 1981.

[12] S. Sinha and B. Schunck, A Two Stage Algorithm for Discontinuity-Preserving Surface Reconstruction, IEEE Trans. on Pattern Analysis and Machine Intelligence, Vol.14, No.1, pp.36-55, 1992.

[13] S. Sun, M. B. Egerstedt, and C. F. Martin, Control theoretic smoothing splines, IEEE Trans. Automat. Control, 45, no. 12, pp.2271-2279, 2000

[14] K. Takayama and H. Kano: A New Approach to Synthesizing Free Motions of Robotic Manipulators Based on a Concept of Unit Motions, IEEE Trans. SMC., Vol. 25, No. 3, pp. 453-463, March, 1995.

[15] G. Wahba, Spline models for observational data CBMS-NSF Regional Conference Series in Applied Mathematics, 59, Society for Industrial and Applied Mathematics (SIAM), Philadelphia, PA, 1990.

[16] Z. Zhang, J. Tomlinson and C. Martin, Splines and Linear Control Theory, Acta Applicandae Mathematicae, 49, pp.1-34, 1997. 